55 years old; $P<0.001$ for both comparisons). Multivariate analysis showed that the presence of patent foramen ovale was independently associated with cryptogenic stroke in both the older age-group (adjusted odds ratio 3.00, 95\% Cl 1.73-5.23; $P<0.001)$ and the younger age-group (odds ratio $3.70,95 \% \mathrm{Cl} 1.42-9.65$; $P=0.008$ ). Unadjusted univariate analysis revealed that the presence of concomitant atrial septal aneurysm strengthened the association between patent foramen ovale and cryptogenic stroke in both age-groups.

Original article Handke $\mathrm{M}$ et al. (2007) Patent foramen ovale and cryptogenic stroke in older patients. N Engl J Med 357: $2262-2268$

\section{Childhood BMI is associated with adult risk of heart disease}

Childhood obesity rates are growing, but the impact of this epidemic on the population's subsequent health as adults remains unclear.

Baker et al. investigated the relationship between BMI in childhood and coronary heart disease (CHD) in adulthood. Data were obtained for 276,835 individuals born in Denmark over a 46-year period-yielding a total of 5,063,622 person-years of follow-up. A total of 10,235 CHD events (fatal or nonfatal) were recorded among men and 4,318 among women.

For boys, the likelihood of experiencing a $\mathrm{CHD}$ event when older than 25 years increased significantly with every 1-unit increase in BMI $z$ score recorded at age $7-13$ years. This relationship was linear, and it persisted when adjusted for birth weight. CHD risk also increased with the age of the child, such that the risk associated with a 1-unit increase in $\mathrm{BMI} z$ score at the age of 13 years was almost double that associated with a 1-unit increase in BMI $z$ score at the age of 7 years.

Similar trends were observed among girls, although the overall risks for CHD were lower than for boys. In this sex the association between a 1-unit increase in BMl $z$ score and an increased risk of adult CHD was also linear, but it only reached significance when BMI was measured in girls 10-13 years old.

This study, comprising a very large cohort, demonstrates that $\mathrm{BMI}$ in childhood relates directly to CHD risk in adulthood. Intervention to help children maintain a healthy weight could be warranted.

Original article Baker JL et al. (2007) Childhood body-mass index and the risk of coronary heart disease in adulthood. N Engl J Med 357: 2329-2337

\section{Blood transfusion during cardiac surgery: more harm than good?}

It remains unclear whether the benefits of red blood cell (RBC) transfusion during cardiac surgery outweigh the risks. A retrospective analysis of data from UK registries has provided further insights into this issue.

Among adults who underwent cardiac surgery between 1 April 1996 and 31 December 2003,737 of 8,516 patients subsequently developed composite infections and 832 of 8,518 patients experienced composite ischemic outcomes. Analysis of these data showed that the risk of infection was considerably higher among those who had received an RBC transfusion than among those who had not (adjusted odds ratio $3.38,95 \% \mathrm{Cl} 2.60-4.40$ ). In addition, transfused patients were at higher risk of composite ischemic outcomes (adjusted odds ratio $3.35,95 \% \mathrm{Cl} 2.68-4.35$ ).

Furthermore, RBC transfusion was associated with longer postoperative hospital stays and increased all-cause mortality - the adjusted hazard ratios for hospital discharge at any postoperative time and death in the 30 days following surgery were $0.63(95 \% \mathrm{Cl} 0.60-0.67)$ and 6.69 (95\% Cl 3.36-15.1), respectively. These associations were reflected in overall hospital costs, which were, on average, 1.42 times higher among patients who received RBC transfusions (95\% Cl 1.37-1.46). For patients who received more than nine RBC units during surgery, the cost increased by an average of 3.35 times (95\% Cl 3.03-3.70).

These findings suggest that RBC transfusion is, in fact, harmful for the majority of patients undergoing cardiac surgery and could be a waste of vital resources.

Original article Murphy GJ et al. (2007) Increased mortality, postoperative morbidity, and cost after red blood cell transfusion in patients having cardiac surgery. Circulation 116: $2544-2552$ 